Research Article

\title{
Mathematical Analysis of the Effects of Controls on the Transmission Dynamics of Anthrax in Both Animal and Human Populations
}

\author{
Elijah B. Baloba $\mathbb{D},{ }^{1}$ Baba Seidu $\mathbb{D}^{2},{ }^{2}$ and Christopher Saaha Bornaa $\mathbb{D}^{3}$ \\ ${ }^{1}$ Gowrie Senior High/Technical School, Department of Mathematics, P.O. Box 76, Bolgatanga, UE/R, Ghana \\ ${ }^{2}$ Department of Mathematics, Faculty of Mathematical Sciences, University for Development Studies, Navrongo Campus, \\ P.O. Box 24, Navrongo, UE/R, Ghana \\ ${ }^{3}$ Department of Science and Mathematics Education, Faculty of Mathematical Sciences, University for Development Studies, \\ Navrongo Campus, P.O. Box 24, Navrongo, UE/R, Ghana \\ Correspondence should be addressed to Elijah B. Baloba; balobaelijah@gmail.com
}

Received 20 July 2019; Revised 15 October 2019; Accepted 23 January 2020; Published 18 February 2020

Academic Editor: Konstantin Blyuss

Copyright (c) 2020 Elijah B. Baloba et al. This is an open access article distributed under the Creative Commons Attribution License, which permits unrestricted use, distribution, and reproduction in any medium, provided the original work is properly cited.

\begin{abstract}
A nonlinear differential equation model is proposed to study the impact of vaccination on the transmission dynamics of anthrax in both livestock and human populations. The model is shown to exhibit only two equilibria, namely, the disease-free and the endemic equilibrium points, which are proven to be locally stable if the basic reproduction number $\left(\mathscr{R}_{0}\right)$ is less than unity and greater than unity, respectively. Local sensitivity analysis shows that the infection rate, pathogen-shedding rate, and rate of vaccination of livestock are parameters with the most positive impact on disease spread, whereas the rate of disinfection followed by the rate of vaccination are the parameters with the most negative impact on disease spread. Numerical simulation shows that implementing all control measures (i.e., vaccination, education, disinfection, and treatment) is a most effective strategy to curb disease spread.
\end{abstract}

\section{Introduction}

Poverty is one of the major challenges facing the developing world, and household income is seen as one of the factors worsening poverty situations for many developing countries, where hunger and extreme poverty are widespread [1]. Livestock production has therefore become a key option for policy makers and stakeholders for increasing income and improving livelihood of rural households $[2,3]$. The livestock sector does not only provide manure for crop and vegetable farmers [4] but also provides food and nutritional security, financial security, and employment [3] to improve the standard of living, especially in rural households. Over 100,000 households in Ghana depend on livestock production for their livelihood, especially in the five regions of northern Ghana [5]. Globally, about 70\% of the world's 1.4 billion extremely poor people depend on livestock [6]. One of the setbacks of livestock rearing is the anthrax disease which mainly affects livestock and other herbivores [7]. Anthrax as an infection caused by the bacterium, Bacillus anthracis, is endemic throughout Africa and other parts of the world [8]. Anthrax results in considerable livestock and wildlife losses and also causes severe and fatal infections in the human population [8]. There has not been any reported case of human-to-human anthrax transmission [9], but it is one of the diseases causing poverty and negatively affecting both livelihoods and human health with serious implications [10].

Anthrax occurs every year, leading to deaths of many animals ranging from hundreds to thousands and 
transmission to humans. Humans often contract the disease from an exposure to infected animals or their products $[8,11]$. It is estimated that about 2,000 to 20,000 human cases of anthrax occur each year worldwide [11].

Ghana has 28 scheduled livestock diseases, of which anthrax is one of the major occurrences in the country and causes severe livestock mortality and deaths in human population. Anthrax occurrence rose from 29 cases in the year 2012 to 1875 cases in 2013 and 943 cases in 2014 [3]. Aside causing human and animal mortality, it has devastating effects on the economy and livelihood systems in Ghana and sub-Sahara Africa since it affects the quality and quantity of meat, milk, and manure output thereby causing food insecurity, especially in poor rural households [3].

The exploration and control of infectious diseases through mathematical modeling has proved to be an important tool for researchers as it helps in increasing our understanding of infectious diseases by revealing some of the complex dynamics of the diseases being modeled [12]. For anthrax disease, some mathematical models (e.g., [13-17]) have been proposed in the literature to help improve the understanding of how the disease spreads and can be eradicated.

In a study by Hahn and Furniss [13], a deterministic model of an anthrax epizootic was studied with the hypothesis that anthrax is transmitted through environmental contamination. An analytic form of the generation multiplication rate was established as well as an analytical expression for the threshold of the disease.

Friedman and Yakubu [14] extended the model of Hahn and Furniss [13] by studying the effects of anthrax transmission, carcass ingestion, carcass-induced environmental contamination and migration rates on the persistence or extinction of animal population. They showed that decreasing the levels of carcass ingestion may not always lead to a reduction in the number of infective animals but increasing the rate of carcass-induced environmental contamination can result in extinction of a persistent animal population.

Mushayabasa [15] also extended the model of Hahn and Furniss [13] by including a fixed time delay and environmental decontamination on eradication of anthrax disease. They explored the effectiveness of environmental decontamination on eradication of anthrax disease through the reproduction number.

Sinkie and Murthy [16] extended the model of Mushayabasa [15] by assuming that some infective animals can display clinical signs of the disease and can be cured by treatment. Their study showed that the pathogen level decreased and susceptible animals increased when the curing rate is increased through treatment.

Osman et al. [18] extended the model of Friedman and Yakubu [14] by constructing a mathematical model of anthrax in human and animal population to investigate the impact of vaccination through sensitivity analysis.

Whilst so much research has been done on zoonotic diseases and anthrax in particular, it is worth noting that not much has been done in the use of Mathematics to model the impact of vaccination and education on the transmission dynamics of anthrax disease in both human and animal populations. In this paper, the impact of anthrax on food security, income, and the health of human beings have necessitated investigation of the transmission and control of anthrax disease in livestock and human populations through mathematical modeling by extending the study of Sinkie and Murthy [16]. This was done by incorporating vaccination of susceptible livestock, cross contamination of the human population, and including education of the human population as a control measure.

\section{Model Formulation}

In this section, a deterministic model is proposed for the study of anthrax transmission in both livestock and human populations in the presence of vaccination programme and education. The following assumptions are considered:

(i) Anthrax is spread by environmental contamination

(ii) There is no vertical transmission

(iii) Susceptible livestock get infected only through ingestion of the anthrax pathogens

(iv) Some infected livestock can show symptoms of the disease and can be cured through treatment

(v) Susceptibles, infectives, educated population, and vaccinated livestock can exit naturally

(vi) Newly born and other susceptible livestock are vaccinated

(vii) Susceptible humans get infected by coming into contact with infected livestock, infected carcass, and pathogens

(viii) There is no human-to-human infection.

The compartmental diagram in Figure 1 shows the transmission of anthrax in both humans and livestock population. The livestock population is subdivided into three mutually exclusive compartments of susceptible livestock $S_{\mathrm{L}}(t)$, vaccinated $V_{\mathrm{L}}(t)$, and infected individuals $I_{\mathrm{L}}(t)$, with adjoining compartments of infected carcass $C(t)$ and pathogens $P_{\mathrm{L}}(t)$ shed into the environment. The livestock that are at risk of getting the infection are called the susceptible livestock. Vaccinated livestock are those that received vaccination before the outbreak of the disease. Infected livestock include those that show symptoms of the anthrax disease. 


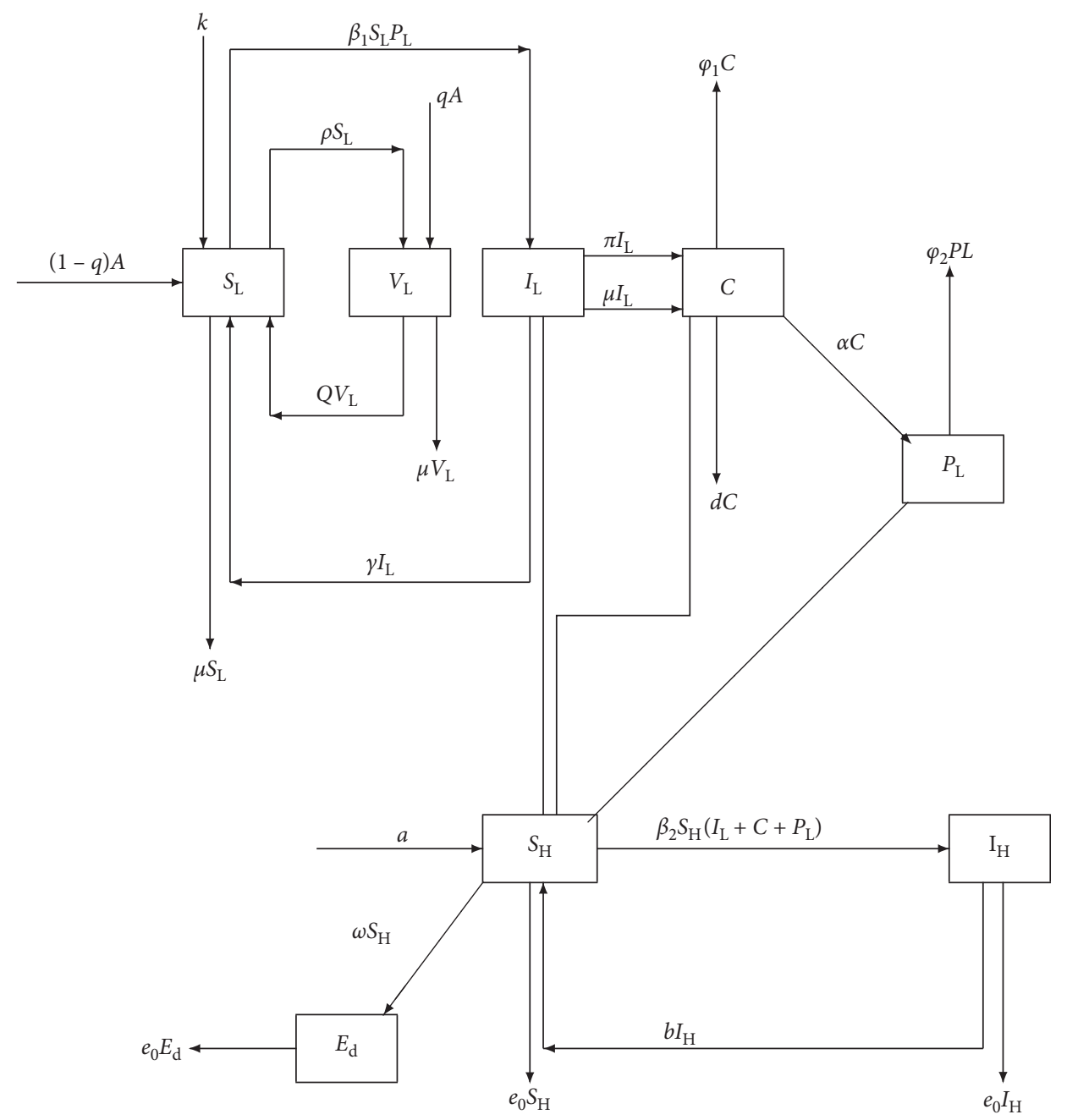

Figure 1: Schematic diagram of anthrax epidemic in both livestock and human populations.

The spillover compartmental model for the human population consists of the susceptible human compartment $S_{\mathrm{H}}(t)$, who are at risk of contracting the anthrax disease upon contact with the infected animal, infected carcass, or pathogen in the environment. A portion $E_{\mathrm{d}}$ of the human population is a compartment of careful susceptible human population who cannot contract the disease due to education or experience. The infected human compartment $I_{\mathrm{H}}(t)$ includes individuals who show symptoms of the disease.

Table 1 summarizes the definition of parameters and variables used in the model.

The susceptible livestock in compartment $S_{\mathrm{L}}$ gets infected when they ingest or come into contact with pathogens in compartment $P_{\mathrm{L}}$ at rate $\beta_{1}$. Livestock that show symptoms of the disease are treated to recover without immunity at rate $\gamma$ to become susceptible.

A fraction, $q$, of newborns (recruits) $A$ are vaccinated and thus are placed in compartment $V_{\mathrm{L}}$. That is, $q A$ livestock enters compartment $V_{\mathrm{L}}$ while the rest of newborns $(1-q) A$ enter compartment $S_{\mathrm{L}}$. Susceptible livestock are vaccinated at rate $\rho$ to join compartment, $V_{\mathrm{L}}$. It is assumed that the vaccinated individuals have temporary immunity so that $Q$ is the rate at which vaccinated individuals lose immunity and return to compartment $S_{\mathrm{L}}$.

Infected carcasses of livestock are placed in compartment $C$ and are disinfected at a rate, $\phi_{1}$. The remaining pathogens from the carcasses get into the environment at a rate $\alpha$ to form compartment $P_{\mathrm{L}}$. The environment is disinfected of pathogens at a rate of $\phi_{2}$.

Humans can get the disease by consuming infected animal products or coming into contact with anthrax infected carcasses. In addition, it is assumed that human beings can acquire anthrax directly by getting into contact with free living spores or pathogens in the environment. As a means of controlling the disease, education is assumed to lead susceptible humans to gain knowledge at rate $\omega$ to avoid being infected. The model under discussion is thus represented by the following system of equations: 
Table 1: Parameters used in the model.

\begin{tabular}{|c|c|c|c|}
\hline Par. & Description & Value & References \\
\hline$\beta_{1}$ & Infection rate & 0.02 & \\
\hline$K$ & The rate of inflow of animals & 0.99 & {$[16]$} \\
\hline$\gamma$ & The recovery rate of infected animals & 0.0025 & [18] \\
\hline$\mu$ & Natural death rate & 0.0001 & {$[16]$} \\
\hline$\pi$ & Anthrax-induced death rate & 0.5 & [16] \\
\hline$\phi_{1}$ & The rate of disinfection of infected carcass & 0.6 & Assumed \\
\hline$\phi_{2}$ & The rate of disinfection of pathogens in the environment & 0.5 & [16] \\
\hline$d$ & Decay rate of infective carcasses & 0.8 & {$[16]$} \\
\hline$\alpha$ & The rate of shedding pathogens into the ground & 0.45 & [18] \\
\hline$A$ & Rate of inflow of livestock & 0.75 & Assumed \\
\hline$q$ & Fraction of recruited livestock that are vaccinated & 0.7 & Assumed \\
\hline Q & Rate of loss of immunity & 0.002 & Assumed \\
\hline$a$ & Recruitment rate of humans & 0.92 & \\
\hline$e_{0}$ & Natural death rate in humans & 0.0001 & \\
\hline$\beta_{2}$ & Infection rate of susceptible humans & 0.0001 & \\
\hline$b$ & The recovering rate of infected humans & 0.04 & \\
\hline$\omega$ & Rate at which humans acquire knowledge to avoid infection & 0.50 & \\
\hline$\rho$ & Rate of vaccination of susceptibles & 0.75 & Assumed \\
\hline$S_{\mathrm{L}}$ & Population size of susceptible livestock & \multicolumn{2}{|c|}{ State variable } \\
\hline$V_{\mathrm{L}}$ & Population size of vaccinated livestock & \multicolumn{2}{|c|}{ State variable } \\
\hline$I_{\mathrm{L}}$ & Population size of infected livestock & \multicolumn{2}{|c|}{ State variable } \\
\hline$C$ & Population size of carcass of livestock & \multicolumn{2}{|c|}{ State variable } \\
\hline$P_{\mathrm{L}}$ & Concentration of anthrax pathogens in the environment & \multicolumn{2}{|c|}{ State variable } \\
\hline$S_{\mathrm{H}}$ & Population size of susceptible humans & \multicolumn{2}{|c|}{ State variable } \\
\hline E & Population size of humans educated enough to avoid infection & \multicolumn{2}{|c|}{ State variable } \\
\hline$I_{\mathrm{H}}$ & Population size of infected humans & \multicolumn{2}{|c|}{ State variable } \\
\hline
\end{tabular}

$\frac{\mathrm{d} S_{L}}{\mathrm{~d} t}=k+(1-q) A+Q V_{\mathrm{L}}(t)+\gamma I_{\mathrm{L}}(t)-\left(\beta_{1} P_{\mathrm{L}}(t)+\mu+\rho\right) S_{\mathrm{L}}(t)$

$\frac{\mathrm{d} V_{\mathrm{L}}}{\mathrm{d} t}=\rho S_{\mathrm{L}}(t)+q A-(Q+\mu) V_{\mathrm{L}}(t)$,

$\frac{\mathrm{d} I_{\mathrm{L}}}{\mathrm{d} t}=\beta_{1} S_{\mathrm{L}}(t) P_{\mathrm{L}}(t)-(\gamma+\pi+\mu) I_{\mathrm{L}}(t)$,

$\frac{\mathrm{d} C}{\mathrm{~d} t}=(\pi+\mu) I_{\mathrm{L}}(t)-\left(\phi_{1}+d\right) C(t)$,

$\frac{\mathrm{d} P_{\mathrm{L}}}{\mathrm{d} t}=\alpha C(t)-\phi_{2} P_{\mathrm{L}}(t)$

$\frac{\mathrm{d} S_{\mathrm{H}}}{\mathrm{d} t}=a+b I_{\mathrm{H}}(t)-\left[\beta_{2}\left(I_{\mathrm{L}}(t)+C(t)+P_{\mathrm{L}}(t)\right)+\omega+e_{0}\right] S_{\mathrm{H}}(t)$,

$\frac{\mathrm{d} E_{\mathrm{d}}}{\mathrm{d} t}=\omega S_{\mathrm{H}}(t)-e_{0} E_{\mathrm{d}}(t)$,

$\frac{\mathrm{d} I_{\mathrm{H}}}{\mathrm{d} t}=\beta_{2}\left(I_{\mathrm{L}}(t)+C(t)+P_{\mathrm{L}}(t)\right) S_{\mathrm{H}}(t)-\left(b+e_{0}\right) I_{\mathrm{H}}(t)$

with initial conditions $S_{\mathrm{L}}(0) \geq 0, V_{\mathrm{L}}(0) \geq 0, I_{\mathrm{L}}(0) \geq 0, C(0) \geq 0$,

$P_{\mathrm{L}}(0) \geq 0, S_{\mathrm{H}}(0) \geq 0, E_{\mathrm{d}}(0) \geq 0, I_{\mathrm{H}}(0) \geq 0$.

For convenience, we use the following substitutions:

$$
\begin{aligned}
\psi_{1} & =k+(1-q) A, \\
\psi_{2} & =\mu+\rho, \\
\psi_{3} & =\gamma+\pi+\mu, \\
\psi_{4} & =\phi_{1}+d, \\
\psi_{5} & =Q+\mu, \\
\psi_{6} & =\omega+e_{0}, \\
\psi_{7} & =\pi+\mu, \\
\Gamma_{1} & =b+e_{0}, \\
\delta & =\beta_{2}\left(I_{\mathrm{L}}+C+P_{\mathrm{L}}\right) .
\end{aligned}
$$

All model parameters are assumed to be nonnegative, and hence, we are only concerned with nonnegative solutions of equation (1).

In the next section, the qualitative analysis of the model is carried out.

\section{Qualitative Analysis}

\subsection{Positivity of Solutions}

Theorem 1. If the initial conditions of the state equations are nonnegative (i.e., $S_{L}(0) \geq 0, V_{L}(0) \geq 0, I_{L}(0) \geq 0, C(0) \geq 0$, $\left.P_{L}(0) \geq 0, S_{H}(0) \geq 0, E_{d}(0) \geq 0, I_{H}(0) \geq 0\right)$, then future solutions are also nonnegative (i.e., $S_{L}(t) \geq 0, V_{L}(t) \geq 0, I_{L}(t) \geq 0$, $C(t) \geq 0, P_{L}(t) \geq 0, S_{H}(t) \geq 0, E_{d}(t) \geq 0$, and $\left.I_{H}(t) \geq 0\right)$.

Proof. Let $t>0$ be the first time such that $S_{\mathrm{L}}(t)=0$. Then, from the first equation, we have $\mathrm{d} S_{\mathrm{L}} / \mathrm{d} t=k+$ $(1-q) A+Q V_{\mathrm{L}}+\gamma I_{\mathrm{L}} \geq 0$. Hence, $S_{\mathrm{L}}(t) \geq \forall t>0$. 
Similarly, consider a positive time $t$. If $V_{\mathrm{L}}(t)=0$, then the second equation becomes $\mathrm{d} V_{\mathrm{L}} / \mathrm{d} t=\rho S_{\mathrm{L}}+q A \geq 0$, which implies that $V_{\mathrm{L}}(t) \geq 0, \forall t>0$. All the remaining state variables can similarly be shown to be nonnegative for all positive times. This concludes the proof.

3.2. Boundedness and Feasible Region of Solutions of Model. The feasible region of the model is the set containing reasonable solutions of the model. In this section, we show that there exists a region within which the model can sufficiently be studied. In this region, the model is epidemiologically and mathematically well posed. This is provided for in the following theorem.

Theorem 2. All solutions of model (1) are uniformly bounded and contained in the feasible region defined by $\xi=$ $\xi_{L} \times \quad \xi_{H}, \forall t>0$, where $\xi_{L}=\left\{\left(S_{L}(t), V_{L}(t), I_{L}(t)\right) \in \mathbb{R}_{+}^{3} \mid\right.$ $\left.\limsup _{t \rightarrow \infty} N_{L} t \leq((k+A) / \mu)\right\}$ and $\xi_{H}=\left\{\left(S_{H}(t), E_{d}(t), I_{H}\right.\right.$ $\left.(t)) \in \mathbb{R}_{+}^{3} \mid \lim \sup _{t \rightarrow \infty} N_{H}(t) \leq\left(a / e_{0}\right)\right\}$. That is the region, $\xi$ is positively invariant under the flow defined by model (1).

Proof. Let the total livestock and human populations be denoted by $N_{\mathrm{L}}$ and $N_{\mathrm{H}}$.

Then $N_{\mathrm{L}}=S_{\mathrm{L}}+V_{\mathrm{L}}+I_{\mathrm{L}}$ and $N_{\mathrm{H}}=S_{\mathrm{H}}+E_{\mathrm{d}}+I_{\mathrm{H}}$, respectively. Then we have $\mathrm{d} N_{\mathrm{L}} / \mathrm{d} t=k+A-\mu N_{\mathrm{L}}-\pi I_{\mathrm{L}}$ and $\mathrm{d} N_{\mathrm{H}} / \mathrm{d} t=a-e_{0} N_{\mathrm{H}}$.

From $\mathrm{d} N_{\mathrm{L}} / \mathrm{d} t$, we have $\mathrm{d} N_{\mathrm{L}} / \mathrm{d} t \leq k+A-\mu N_{\mathrm{L}}$.

By standard comparison theorems [19], we have $N_{\mathrm{L}}(t) \leq N_{\mathrm{L}}(0) e^{-\mu t}+((k+A) / \mu)\left(1-e^{-\mu t}\right), \quad$ so that $\lim \sup _{t \rightarrow \infty} N_{\mathrm{L}}(t) \leq((k+A) / \mu)$.

Also, from $\mathrm{d} N_{\mathrm{H}} / \mathrm{d} t$, we have $N_{\mathrm{H}}(t)=N_{\mathrm{H}}(0) e^{-e_{0} t}+$ $\left(a / e_{0}\right)\left(1-e^{-e_{0} t}\right)$.

Hence all solutions are uniformly bounded.

Now, define

$$
\begin{aligned}
& \xi_{\mathrm{L}}=\left\{\left(S_{\mathrm{L}}(t), V_{\mathrm{L}}(t), I_{\mathrm{L}}(t)\right) \in \mathbb{R}_{+}^{3} \mid \limsup _{t \rightarrow \infty} N_{\mathrm{L}}(t) \leq \frac{k+A}{\mu}\right\}, \\
& \xi_{\mathrm{H}}=\left\{\left(S_{\mathrm{H}}(t), E_{\mathrm{d}}(t), I_{\mathrm{H}}(t)\right) \in \mathbb{R}_{+}^{3} \mid \limsup _{t \rightarrow \infty} N_{\mathrm{H}}(t) \leq \frac{a}{e_{0}}\right\} .
\end{aligned}
$$

Then, the region $\xi=\xi_{\mathrm{L}} \times \xi_{\mathrm{H}}$ is positively invariant under model (1). Therefore, the model is mathematically and epidemiologically well posed, and hence, it is sufficient to study the model in $\xi$. This concludes the proof.

3.3. Equilibrium Points. Model (1) has basically two equilibrium points, namely, the disease-free and endemic equilibrium points. The disease-free equilibrium is given by

$$
\mathscr{E}_{0}=\left(\frac{\psi_{1}}{\psi_{2}}+\frac{Q\left(\rho \psi_{1}+q A \psi_{2}\right)}{\psi_{2}\left(\psi_{2} \psi_{5}-\rho Q\right)}, \frac{\rho \psi_{1}+q A \psi_{2}}{\psi_{2} \psi_{5}-\rho Q}, 0,0,0, \frac{a}{\psi_{6}}, \frac{\omega a}{\psi_{6}}, 0\right) .
$$

Next, we find the basic reproduction number using the method described in [20]. The basic reproduction number is defined as the average number of secondary anthrax infections a single infected animal causes during its infectious life if it is introduced into an initially anthrax-free human livestock system. The basic reproduction number $\mathscr{R}_{0}$ is the dominant eigenvalue (spectral radius) of the next generation matrix $F V^{-1}$, where $F$ and $V$ are the transmission and transition matrices [20]. For our model, the matrices are given by

$$
\begin{aligned}
V & =\left[\begin{array}{cccc}
\psi_{3} & 0 & 0 & 0 \\
-\psi_{7} & \psi_{4} & 0 & 0 \\
0 & -\alpha & \phi_{2} & 0 \\
0 & 0 & 0 & \Gamma_{1}
\end{array}\right], \\
F & =\left[\begin{array}{cccc}
0 & 0 & \beta_{1} S_{\mathrm{L}}(t) & 0 \\
0 & 0 & 0 & 0 \\
0 & 0 & 0 & 0 \\
\beta_{2} S_{\mathrm{H}}(t) & \beta_{2} S_{\mathrm{H}}(t) & \beta_{2} S_{\mathrm{H}}(t) & 0
\end{array}\right] .
\end{aligned}
$$

The basic reproduction number is thus given by

$$
\mathscr{R}_{0}=\frac{\beta_{1} \alpha \psi_{7} S_{\mathrm{L} 0}}{\psi_{3} \psi_{4} \phi_{2}} \text {. }
$$

In the presence of pathogens in the environment, the model exhibits the endemic equilibrium point, $\mathscr{E}^{*}=\left(S_{\mathrm{L}}^{*}, V_{\mathrm{L}}^{*}, I_{\mathrm{L}}^{*}, C^{*}, P_{\mathrm{L}}^{*}, S_{\mathrm{H}}^{*}, E_{\mathrm{d}}^{*}, I_{\mathrm{H}}^{*}\right)$, where

$$
\begin{aligned}
& S_{\mathrm{L}}^{*}=\frac{S_{\mathrm{L} 0}}{\mathscr{R}_{0}} \\
& V_{\mathrm{L}}^{*}=\frac{1}{\psi_{5}}\left(\frac{\rho S_{\mathrm{L} 0}}{\mathscr{R}_{0}}+q A\right), \\
& I_{\mathrm{L}}^{*}=\frac{\mu\left(Q+\psi_{2}\right) S_{\mathrm{L} 0}}{\psi_{5}^{2} \psi_{7}}\left(1-\frac{1}{\mathscr{R}_{0}}\right), \\
& C^{*}=\frac{\mu\left(Q+\psi_{2}\right) S_{\mathrm{L} 0}}{\psi_{4} \psi_{5}^{2}}\left(1-\frac{1}{\mathscr{R}_{0}}\right), \\
& P_{L}^{*}=\frac{\alpha \mu\left(Q+\psi_{2}\right) S_{\mathrm{L} 0}}{\phi_{2} \psi_{4} \psi_{5}^{2}}\left(1-\frac{1}{\mathscr{R}_{0}}\right)
\end{aligned}
$$$$
S_{\mathrm{H}}^{*}=\frac{a \Gamma_{1}}{\Gamma_{1} \psi_{6}+e_{0} \beta_{2}\left(I_{\mathrm{L}}^{*}+C^{*}+P_{\mathrm{L}}^{*}\right)},
$$$$
E_{\mathrm{d}}^{*}=\frac{\omega}{e_{0}} S_{\mathrm{H}}^{*}
$$$$
I_{\mathrm{H}}^{*}=\frac{\beta_{2}\left(I_{\mathrm{L}}^{*}+C^{*}+P_{\mathrm{L}}^{*}\right) S_{\mathrm{H}}^{*}}{\Gamma_{1}},
$$$$
I_{\mathrm{L}}^{*}+C^{*}+P_{\mathrm{L}}^{*}=\frac{\mu\left(Q+\psi_{2}\right) S_{\mathrm{L} 0}}{\psi_{5}^{2} \phi_{2} \psi_{4} \psi_{7}}\left(\phi_{2} \psi_{4}+\left(\alpha+\phi_{2}\right) \psi_{7}\right)\left(1-\frac{1}{\mathscr{R}_{0}}\right),
$$

Clearly, the endemic equilibrium exists only when $\mathscr{R}_{0}>0$. In the next section, we study the local stability of the equilibrium points of the model. 
3.4. Stability Analysis of Equilibrium Points. In this section, we study the local stability of the disease-free and endemic equilibrium points using the indirect Lyapunov method. By this method, an equilibrium point $x^{*}$ of a model $\mathrm{d} x / \mathrm{d} t=$ $f(x)$ is said to be locally asymptotically stable if all eigenvalues of the Jacobian of the model evaluated at $x^{*}$ have negative real parts.

The Jacobian of model (1) is given by

$$
J=\left[\begin{array}{cccccccc}
-\beta_{1} P_{\mathrm{L}}-\psi_{2} & Q & \gamma & 0 & -\beta_{1} S_{\mathrm{L}} & 0 & 0 & 0 \\
\rho & -\psi_{5} & 0 & 0 & 0 & 0 & 0 & 0 \\
\beta_{1} P_{\mathrm{L}} & 0 & -\psi_{3} & 0 & \beta_{1} S_{\mathrm{L}} & 0 & 0 & 0 \\
0 & 0 & \psi_{7} & -\psi_{4} & 0 & 0 & 0 & 0 \\
0 & 0 & 0 & \alpha & -\phi_{2} & 0 & 0 & 0 \\
0 & 0 & -\beta_{2} S_{\mathrm{H}} & -\beta_{2} S_{\mathrm{H}} & -\beta_{2} S_{\mathrm{H}} & -\beta_{2} \delta-\psi_{6} & 0 & b \\
0 & 0 & 0 & 0 & 0 & \omega & -e_{0} & 0 \\
0 & 0 & \beta_{2} S_{\mathrm{H}} & \beta_{2} S_{\mathrm{H}} & \beta_{2} S_{\mathrm{H}} & \beta_{2} \delta & 0 & -\Gamma_{1}
\end{array}\right] .
$$

The Jacobian, $J$, evaluated at $\mathscr{E}_{0}$ has eigenvalues $-e_{0},-\psi_{6}$, and $-\Gamma_{1}$, and the rest satisfy the following characteristic polynomial equation.

where

$$
\begin{aligned}
& k_{0}=\mu \phi_{2} \psi_{4} \psi_{3}\left(Q+\psi_{2}\right)\left(1-\mathscr{R}_{0}\right), \\
& k_{1}=\phi_{2} \psi_{3} \psi_{4}\left(\psi_{5}+\psi_{2}\right)\left(1-\mathscr{R}_{0}\right)+\mu\left(\psi_{4} \psi_{3}+\phi_{2} \psi_{4}+\phi_{2} \psi_{3}\right)\left(Q+\psi_{2}\right), \\
& k_{2}=\phi_{2} \psi_{4} \psi_{3}\left(1-\mathscr{R}_{0}\right)+\left(\psi_{5}+\psi_{2}\right)\left(\psi_{4} \psi_{3}+\phi_{2}\left(\psi_{4}+\psi_{3}\right)\right)+\mu\left(\phi_{2}+\psi_{3}+\psi_{4}\right)\left(Q+\psi_{2}\right), \\
& k_{3}=\left(\psi_{2}+\psi_{4}+\psi_{3}+\psi_{5}\right) \phi_{2}+\psi_{4}\left(\psi_{3}+\psi_{5}+\psi_{2}\right)+\psi_{3}\left(\psi_{5}+\psi_{2}\right)+\mu\left(Q+\psi_{2}\right), \\
& k_{4}=\phi_{2}+\psi_{2}+\psi_{3}+\psi_{4}+\psi_{5} .
\end{aligned}
$$

Clearly, $k_{3}>0, k_{4}>0$ and also $k_{0} \geq 0, k_{1}>0, k_{2}>0$ whenever $\mathscr{R}_{0} \leq 1$.

By the Descartes rule of signs, it can be shown that equation (9) has all its roots with real parts lying in the left half of the plane whenever $\mathscr{R}_{0} \leq 1$. The following result is thus established.

Theorem 3. The disease-free equilibrium point $\mathscr{E}_{0}$ is locally asymptotically stable whenever $\mathscr{R}_{0} \leq 1$ and unstable otherwise.
Also, the Jacobian evaluated at the endemic equilibrium $-e_{0}$ as an eigenvalue, and the remaining eigenvalues can be shown to satisfy the following characteristic polynomial:

$$
\lambda^{7}+\eta_{6} \lambda^{6}+\eta_{5} \lambda^{5}+\eta_{4} \lambda^{4}+\eta_{3} \lambda^{3}+\eta_{2} \lambda^{2}+\eta_{1} \lambda+\eta_{0}=0
$$

where

$$
\begin{aligned}
\eta_{6}= & \Gamma_{1}+\beta_{1} P_{\mathrm{L}}^{*}+\beta_{2} \delta+\phi_{2}+\psi_{2}+\psi_{3}+\psi_{4}+\psi_{5}+\psi_{6}, \eta_{5} \\
= & \left(\beta_{2} \delta+\Gamma_{1}+\psi_{7}+\psi_{5}+\psi_{6}+\phi_{2}+\psi_{4}\right) \beta_{1} P_{\mathrm{L}}^{*} \\
& +\left(\psi_{5}+\psi_{2}+e_{0}+\phi_{2}+\psi_{4}+\psi_{3}\right) \delta \beta_{2}+\left(\phi_{2}+\psi_{6}+\psi_{2}+\psi_{4}+\psi_{3}+\psi_{5}\right) \Gamma_{1}+\left(\psi_{3}+\psi_{6}+\psi_{2}+\psi_{4}+\psi_{5}\right) \phi_{2}+\psi_{3} \psi_{5}+\psi_{3} \psi_{2} \\
& +\psi_{6} \psi_{4}+\psi_{6} \psi_{3}+\psi_{6} \psi_{5}+\psi_{6} \psi_{2}+\psi_{4} \psi_{2}+\mu\left(Q+\psi_{2}\right)+\psi_{4} \psi_{5}+\psi_{4} \psi_{3}, \\
\eta_{4}= & \beta_{1} P_{\mathrm{L}}^{*}\left(\psi_{4}+\psi_{5}+\psi_{7}+\phi_{2}\right)\left(\beta_{2} \delta+\Gamma_{1}+\psi_{6}\right)+\mu\left(\psi_{2}+Q\right)\left(\beta_{2} \delta+\psi_{6}+\phi_{2}+\Gamma_{1}+\psi_{3}+\psi_{4}\right)+\frac{a \Gamma_{1}}{S_{\mathrm{H}}^{*}} \\
& +\left(\psi_{4}+\psi_{5}+\phi_{2}+\beta_{1} P_{\mathrm{L}}^{*}+\psi_{2}+\psi_{3}\right)+\left(\psi_{5}+\psi_{2}\right)\left(\phi_{2}+\psi_{3}+\psi_{4}\right)\left(\beta_{2} \delta+\Gamma_{1}+\psi_{6}\right)+P_{\mathrm{L}}^{*} \beta_{1}\left(\phi_{2} \psi_{5}+\psi_{4} \psi_{5}+\phi_{2} \psi_{4}+\psi_{4} \psi_{7}\right. \\
& \left.+\psi_{5} \psi_{7}+\phi_{2} \psi_{7}\right)+\left(\psi_{4} \psi_{3}+\phi_{2} \psi_{4}+\phi_{2} \psi_{3}\right)\left(\Gamma_{1}+\beta_{2} \delta+\psi_{5}+\psi_{6}+\psi_{2}\right),
\end{aligned}
$$




$$
\begin{aligned}
\eta_{3}= & \frac{a \Gamma_{1}}{S_{\mathrm{H}}^{*}}\left\{\left(\psi_{4}+\psi_{5}+\psi_{7}+\phi_{2}\right) \beta_{1} P_{\mathrm{L}}^{*}+\left(\phi_{2}+\psi_{3}+\mu+\psi_{4}\right) \psi_{2}+\left(\psi_{4}+\psi_{5}+\phi_{2}\right) \psi_{3}+\psi_{4}\left(\phi_{2}+\psi_{5}\right)+\mu Q+\phi_{2} \psi_{5}\right\} \\
& +\left\{\left[\left(\psi_{4}+\psi_{5}+\phi_{2}\right) \psi_{7}+\phi_{2}\left(\psi_{4}+\psi_{5}\right)+\psi_{4} \psi_{5}\right] \beta_{1} P_{\mathrm{L}}^{*}+\left(\psi_{5}+\psi_{2}\right)\left(\psi_{4} \psi_{3}+\phi_{2} \psi_{4}+\phi_{2} \psi_{3}\right)+\mu\left(\phi_{2}+\psi_{3}+\psi_{4}\right)\left(\psi_{2}+Q\right)\right\} \\
& \cdot\left(\beta_{2} \delta+\Gamma_{1}+\psi_{6}\right)+\left(\left(\phi_{2} \psi_{5}+\phi_{2} \psi_{4}+\psi_{4} \psi_{5}\right) \psi_{7}+\phi_{2} \psi_{4} \psi_{5}\right) \beta_{1} P_{\mathrm{L}}^{*}+\mu\left(\psi_{4} \psi_{3}+\phi_{2} \psi_{4}+\phi_{2} \psi_{3}\right)\left(\psi_{2}+Q\right), \\
\eta_{2}= & \frac{a \Gamma_{1}}{S_{\mathrm{H}}^{*}}\left\{\mu\left(\phi_{2}+\psi_{3}+\psi_{4}\right)\left(\psi_{2}+Q\right)+\left(\psi_{5}+\psi_{2}\right)\left(\psi_{4} \psi_{3}+\phi_{2} \psi_{4}+\phi_{2} \psi_{3}\right)+\left[\left(\psi_{4}+\psi_{5}+\phi_{2}\right) \psi_{7}+\left(\psi_{4}+\phi_{2}\right) \psi_{5}+\phi_{2} \psi_{4}\right] \beta_{1} P_{\mathrm{L}}^{*}\right\} \\
& +\beta_{1} \phi_{2} \psi_{4} P_{\mathrm{L}}^{*} \psi_{5} \psi_{7}+\left(\beta_{2} \delta+\Gamma_{1}+\psi_{6}\right)\left\{\left(\left(\phi_{2} \psi_{5}+\phi_{2} \psi_{4}+\psi_{4} \psi_{5}\right) \psi_{7}+\phi_{2} \psi_{4} \psi_{5}\right) \beta_{1} P_{\mathrm{L}}^{*}+\mu\left(\psi_{4} \psi_{3}+\phi_{2} \psi_{4}+\phi_{2} \psi_{3}\right)\left(\psi_{2}+Q\right)\right\}, \\
\eta_{1}= & \beta_{1} P_{\mathrm{L}}^{*}\left(\beta_{2} \delta+\psi_{6}+\Gamma_{1}\right) \phi_{2} \psi_{4} \psi_{5} \psi_{7}+\frac{a \mu \Gamma_{1}}{S_{\mathrm{H}}^{*}}\left(\psi_{4} \psi_{3}+\phi_{2} \psi_{4}+\phi_{2} \psi_{3}\right)\left(\psi_{2}+Q\right)+\frac{a \Gamma_{1}}{S_{\mathrm{H}}^{*}}\left[\left(\psi_{4} \psi_{7}+\psi_{4} \psi_{5}+\psi_{5} \psi_{7}\right) \phi_{2}\right. \\
& \left.+\psi_{5} \psi_{7} \psi_{4}\right] \beta_{1} P_{L}^{*}, \\
\eta_{0}= & \frac{a \Gamma_{1} \phi_{2} \psi_{4}}{S_{\mathrm{H}}^{*}}\left[\beta_{1} \psi_{5} \psi_{7} P_{\mathrm{L}}^{*}+2 \psi_{3} \mu\left(Q+\psi_{2}\right)\right] .
\end{aligned}
$$

Recall that the endemic equilibrium exists only when $\mathscr{R}_{0}>1$ and when that happens, we have $\eta_{i}>0, \forall i=0,1,2, \ldots, 6$. By Descartes's rule of signs, equation (9) has all its roots in the left half of the real plane. This proves that the eigenvalues of the Jacobian of the model evaluated at the endemic equilibrium point (whenever it exists) all have negative real parts. The following results are thus established.

Theorem 4. The endemic equilibrium point $\mathscr{E}^{*}$ is locally asymptotically stable whenever $\mathscr{R}_{0}>1$ and unstable otherwise.

3.5. Local Sensitivity Analysis. In this section, a local sensitivity analysis is conducted on the basic reproduction number using the Normalized Forward Sensitivity Index to determine the impact of parameter values on the basic reproduction number $\mathscr{R}_{0}[21,22]$. The sensitivity indexes in Table 2 were evaluated using the parameter values in Table 1.

\section{Numerical Simulation}

We use the fourth order Runge-Kutta scheme to perform a numerical simulation of the model using parameter values in Table 1. We perform the simulation for different combinations of the controls (vaccination, education, disinfection, and treatment) (see Figures 2-7). We also study the impact of various parameters on model dynamics by running the model for different values of the parameters (see Figures 8-11).

4.1. Findings. The following are the findings of this research:

(1) The disease-free and endemic equilibrium points of the model are stable if the reproduction number is less than one $\left(\mathscr{R}_{0}<1\right)$ and unstable if the reproduction number is greater than one $\left(\mathscr{R}_{0}>1\right)$.

(2) The sensitivity analysis shows that the most sensitive positive parameters are the pathogen-shedding rate, the anthrax infection rate, anthrax-induced death rate, and rate of livestock that lose immunity

(3) The sensitivity analysis also shows that the most sensitive negative parameters are the rate of pathogen disinfection in the environment, the rate of vaccination, and natural death rate. These parameters are inversely related to the reproduction number and indicate that a smaller amount of increment in these parameters will lead to a greater amount of reduction in the basic reproduction number while a smaller amount of decrement will cause a significant increment in the basic reproduction number.

(4) The numerical simulation of the model shows that in the absence of vaccination, the number of infectives will increase drastically.

(5) Further simulation shows that the pathogen level in the environment increases exponential when disinfection is absent.

(6) The most efficient single implementation of the controls in the model is education of susceptible human population.

(7) The most efficient pair of controls are vaccination of susceptible livestock and education of the human population.

(8) The most efficient triple combination of controls is vaccination of susceptible livestock, education of the human population, and disinfection of infected carcass and the pathogen in the environment.

4.2. Results and Discussion. The sensitivity analysis conducted on the basic reproduction number, $\mathscr{R}_{0}$, shows that, the most sensitive positive parameters are the rate of pathogens shed into the environment $(\alpha)$, the rate of anthrax infection (i.e., $\beta_{1}$ ), anthrax-induced death rate $(\pi)$, and the 
TABLE 2: Sensitivity indexes.

\begin{tabular}{lc}
\hline Parameter & Sensitivity index \\
\hline$\phi_{2}$ & -1.0000 \\
$\alpha$ & +1.0000 \\
$\beta_{1}$ & +1.0000 \\
$\pi$ & +0.9998 \\
$\rho$ & -0.9972 \\
$\mu$ & -0.9662 \\
$Q$ & +0.9636 \\
$k$ & +0.5773 \\
$d$ & -0.5333 \\
$\phi_{1}$ & -0.4667 \\
$A$ & +0.4227 \\
$q$ & -0.0146 \\
$\gamma$ & $-7.95 E-04$ \\
\hline
\end{tabular}

rate of animals that lose immunity $(Q)$, and these parameters are directly related to the reproduction number.

Thus, a 10\% increase (or decrease) in the pathogen shedding rate and the rate of infection will result in a $10 \%$ increase (or decrease) in the basic reproduction number, respectively, while a $10 \%$ increase (or decrease) in the anthrax-induced death rate and in the rate of animals that lose immunity will lead to $9.998 \%$ and $9.636 \%$ increase (or decrease) in the basic reproduction number, respectively.

The rate of pathogen disinfection in the environment $\left(\phi_{2}\right)$, the rate of vaccination $(\rho)$, and the natural death rate $(\mu)$ are the most sensitive negative parameters; hence, a $10 \%$ increase (or decrease) in the rate of pathogen disinfection in the environment $\left(\phi_{2}\right)$, the rate of vaccination $(\rho)$, and the natural death rate $(\mu)$ will cause $10 \%, 9.972 \%$, and $9.662 \%$ decrease (or increase) in the basic reproduction number, respectively.

Figure 2 shows a simulation of the basic model when all control measures are absent (that is, $\rho=0, q=0, \phi=0$, and $\gamma=0$ ), and the number of infectives, the carcass, and the pathogen level increase, leading to more infection thus a reduction in the susceptible population. In Figure 3, the number of infectives decreases resulting in a corresponding decrease in the carcass and pathogen levels as more Susceptibles join the vaccinated class. The vaccination increases the population of careful and susceptible humans and also reduces the infective human population. When only education is implemented, it results in high level of careful susceptible humans and livestock populations as well as the overall educated human population as indicated in Figure 4. However, additional measures are required to further reduce the infective populations and the pathogen level.

In Figure 5, a combination of vaccination and education is implemented; this results in low level of infectives of both populations and the number of carcasses but the number of susceptible human population increases. However, the level of pathogens in the environment remains high and therefore requires additional controls.

The number of human and livestock susceptibles and the careful susceptible humans (educated) as well as the vaccinated livestock increase exponentially when all control parameters are implemented, and the infective population for both livestock and humans, the number of carcasses, and the pathogen level decrease as indicated in Figure 6.
Increasing the rate of infection as susceptible humans come into contact with infected livestock, infected carcass, and pathogens in the environment lead to an increment in the number of infective humans as indicated in Figure 7.

It is established from Figure 8 that the susceptible population of both human and livestock increase at smaller values of the infection rate and reduces at higher values. The reduction of susceptible might be as a result of movement from susceptible compartment to the infectious compartment as infection rate increases.

In Figure 9, the vaccinated and educated population increases when the rate of infection is low, and as the rate of infection increases, both vaccinated and educated population reduces. This result shows that it is much safer to vaccinate susceptibles when the infection rate is very lower or if there is not yet an outbreak.

Simulation of susceptible humans at various rates of vaccination in Figure 10 shows that when the vaccination rate is increased the number of human susceptible increases and when the vaccination rate is decreased the susceptible human population also decreases. The vaccinated population increases as the rate of vaccination increases, and when the rate of vaccination is lowered the population decreases.

Finally, the simulation in Figure 11 shows that the human susceptible population is directly related to the education rate, and the rate of education is inversely related to the educated population between time 0 and 4 but above time 4 the rate of education and the educated population are directly related. This simulation shows that the anthrax disease is best controlled in the human population when there is a long-term programme of education on the anthrax disease.

Table 3 shows a summary of the comparison between the controls on each state variable. Here, the control parameters vaccination, education, disinfection, and treatment are ranked as $R 1$ to $R 4$ to determine the most efficient parameter that influence each of the state variables positively. The values from the simulation results are used for this analysis, and the ranks $R 1, R 2, R 3$, and $R 4$ imply first, second, third, and fourth positions, respectively.

In Table 3, the (1st row, 1st column) is $R 3$ and indicates that the vaccination programme is ranked 3 rd in terms of increasing the number of susceptible livestock population when implemented. The (1st row, 3 rd column) is ranked $R 1$ over vaccination, education, and treatment, and this implies that disinfection is ranked first in terms of increasing the number of susceptible livestock when implemented.

The best single control measure for the fight of anthrax within the livestock population is vaccination programme and this is indicated in the (9th row, 1st column) as $R 1$, and the best single control programme for the human population is education and it is indicated in the (10th row, 2nd column) as $R 1$. The overall best single control for the entire model is education of the human population, and it is indicated in the (last row, 2nd column) as 19 (that is, the value with the least sum of ranks). The vaccination programme is ranked first in the following: increasing the vaccinated livestock, decreasing the number of infective livestock, decreasing the number of 


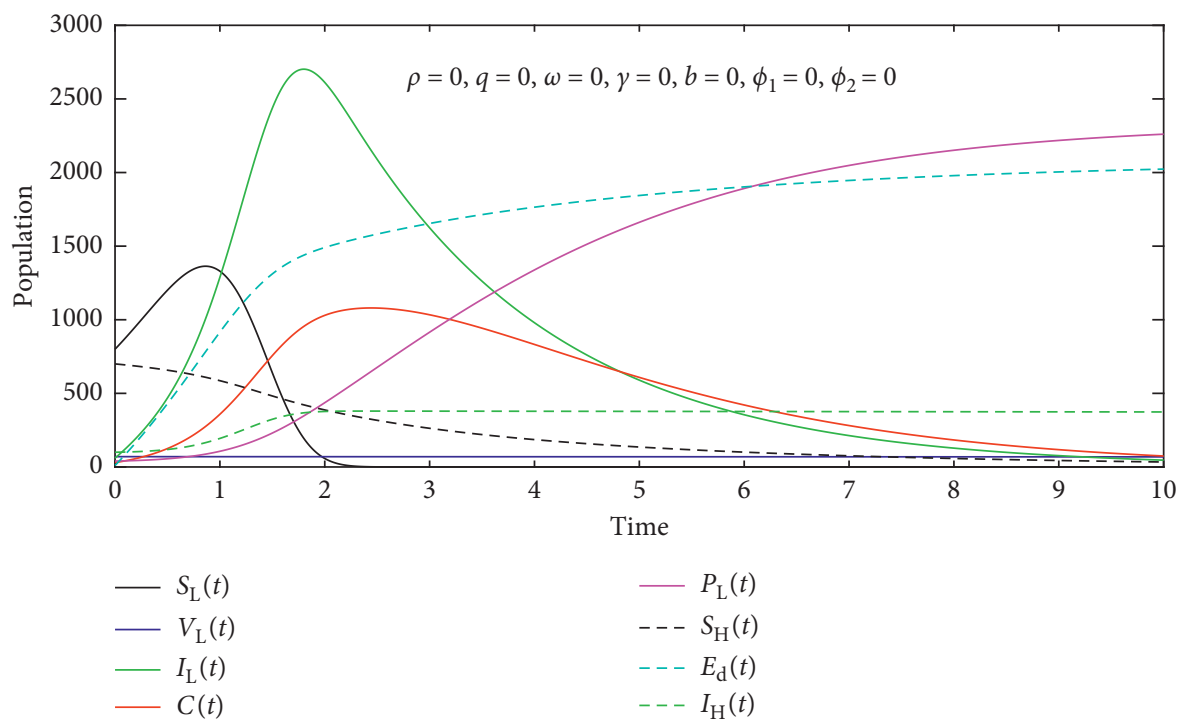

Figure 2: Trajectories of model solution without implementing any control.

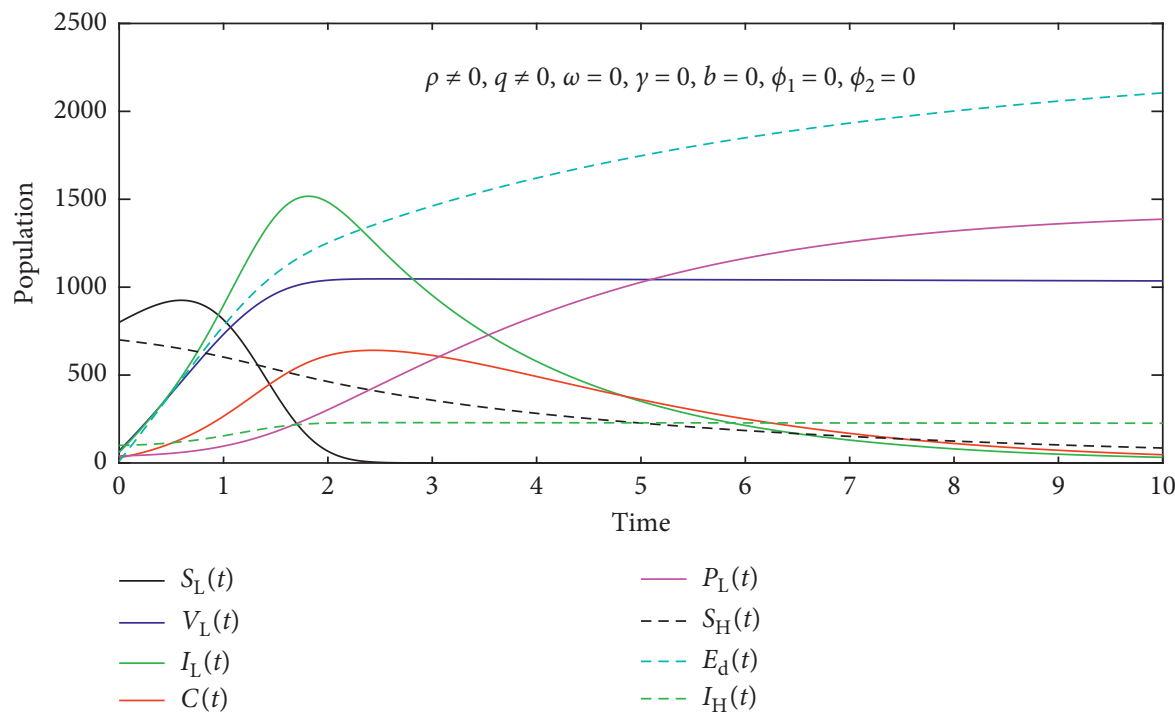

FIGURE 3: Trajectories of model solution when only vaccination is implemented.

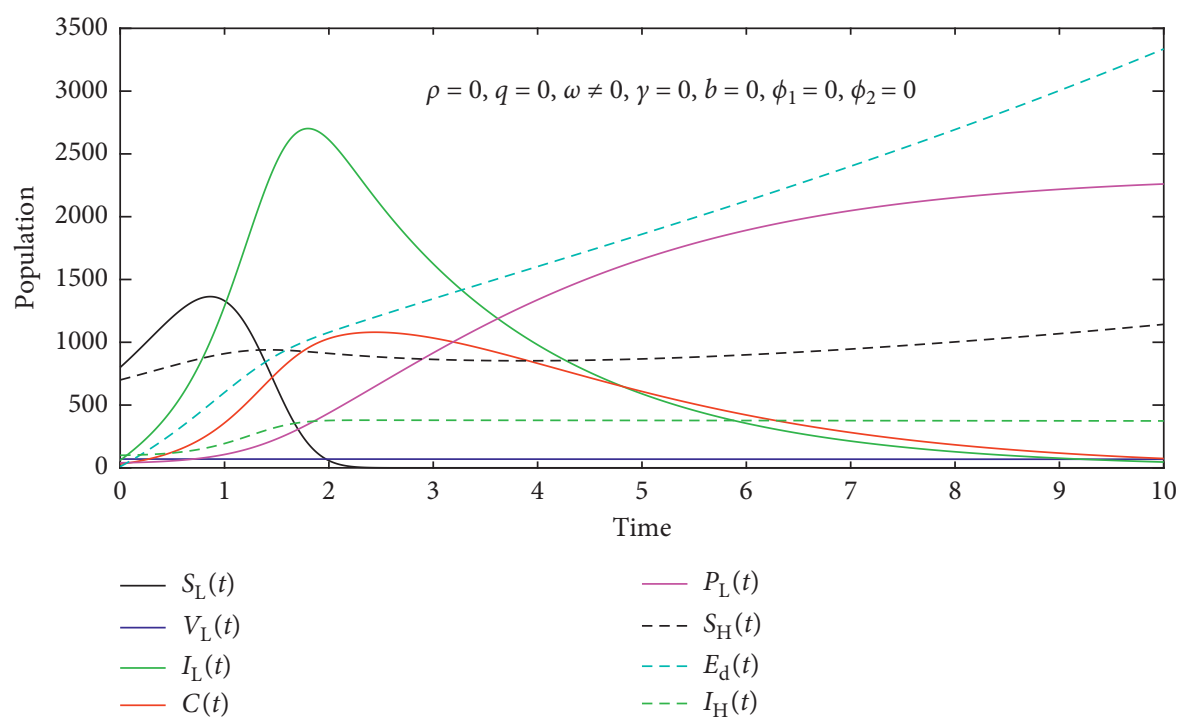

Figure 4: Trajectories of model solution when only education is implemented. 


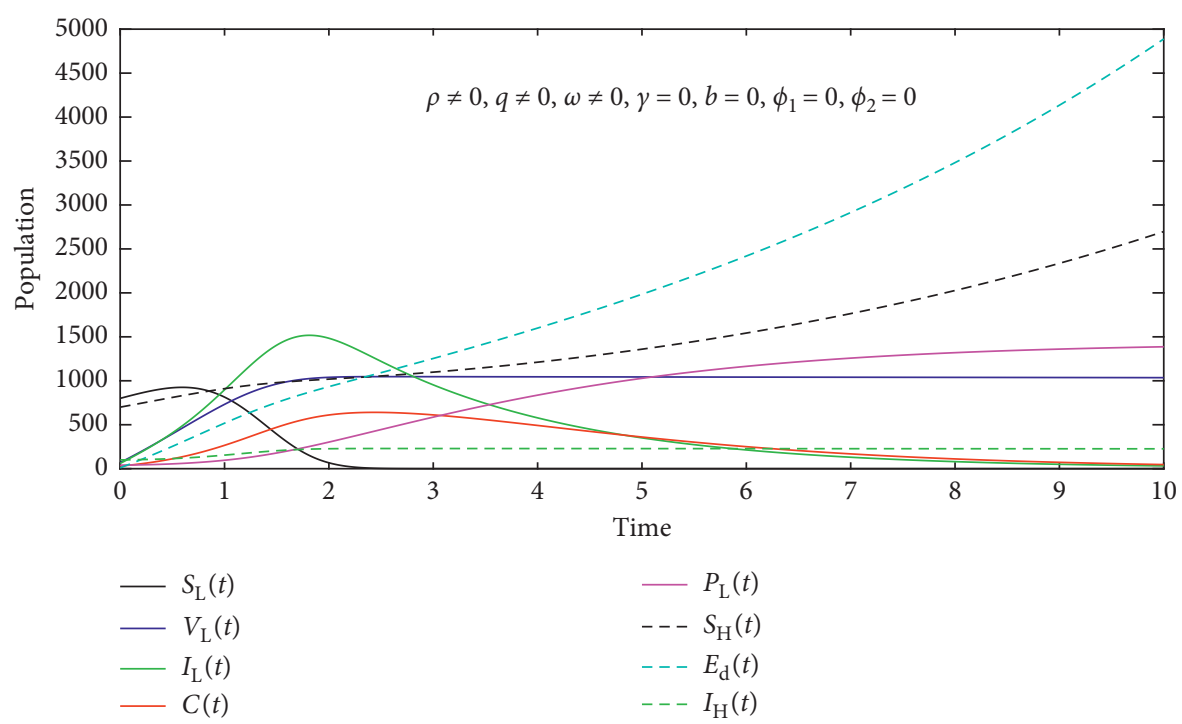

FIGURE 5: Trajectories of model solution when vaccination and education are implemented.

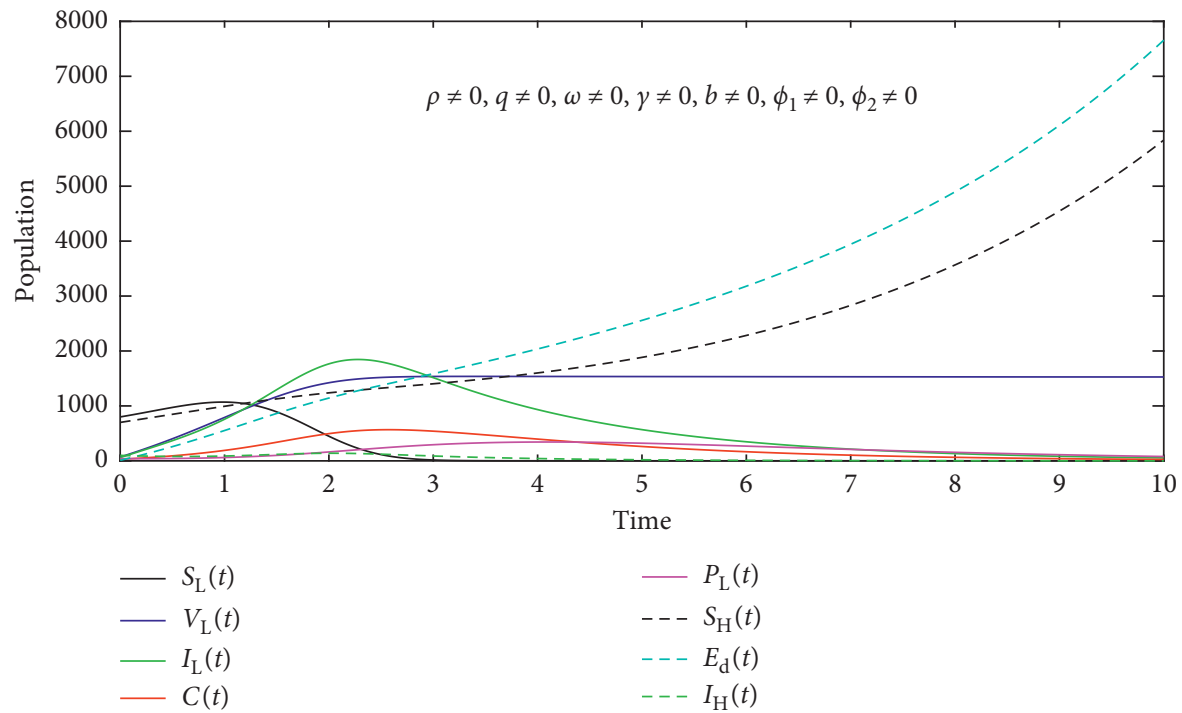

FIgURE 6: Trajectories of model solution when all controls are implemented.

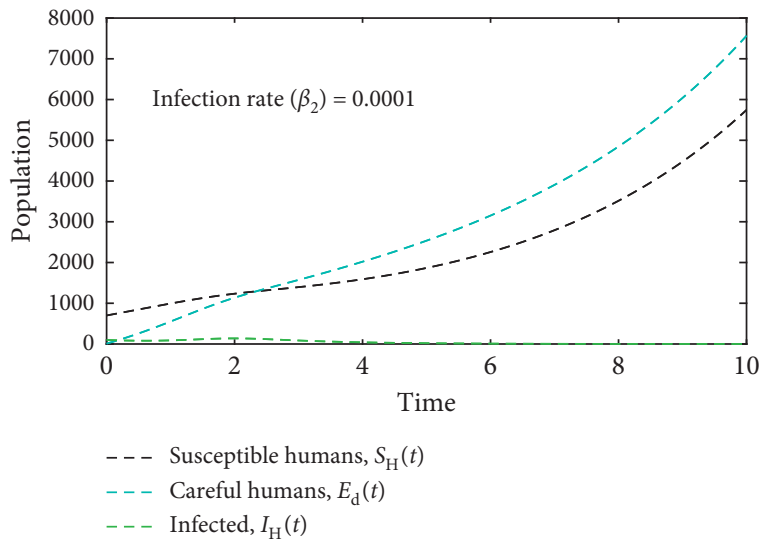

(a)

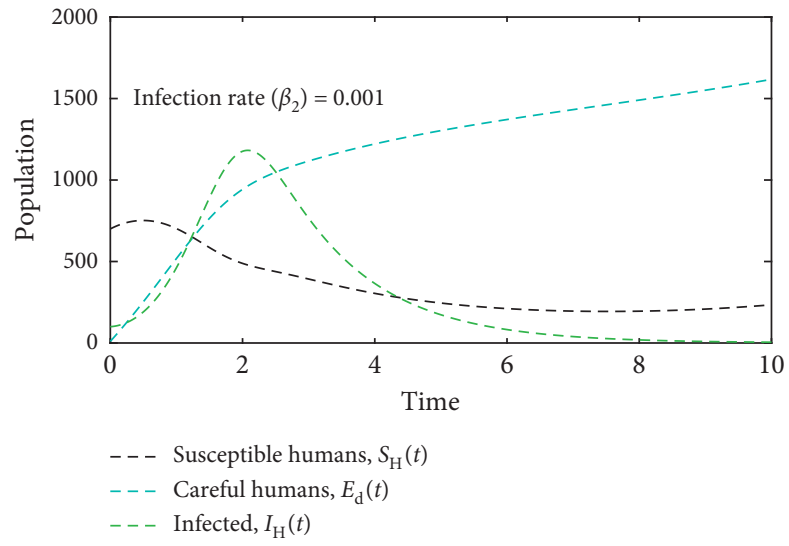

(b)

FIGURE 7: Trajectories of model solution for varying rate of infection. 


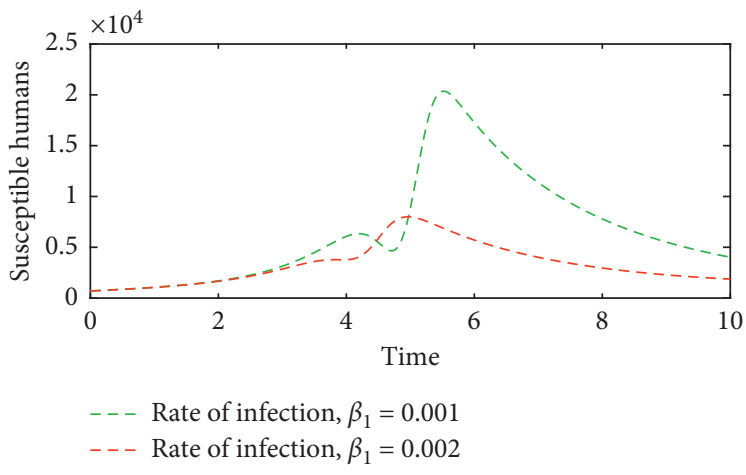

(a)

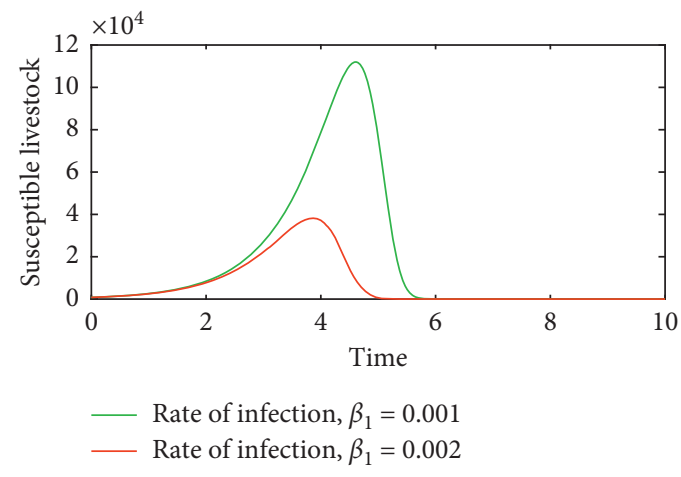

(b)

FIGURE 8: Trajectories of model solution for varying infection rate of livestock.
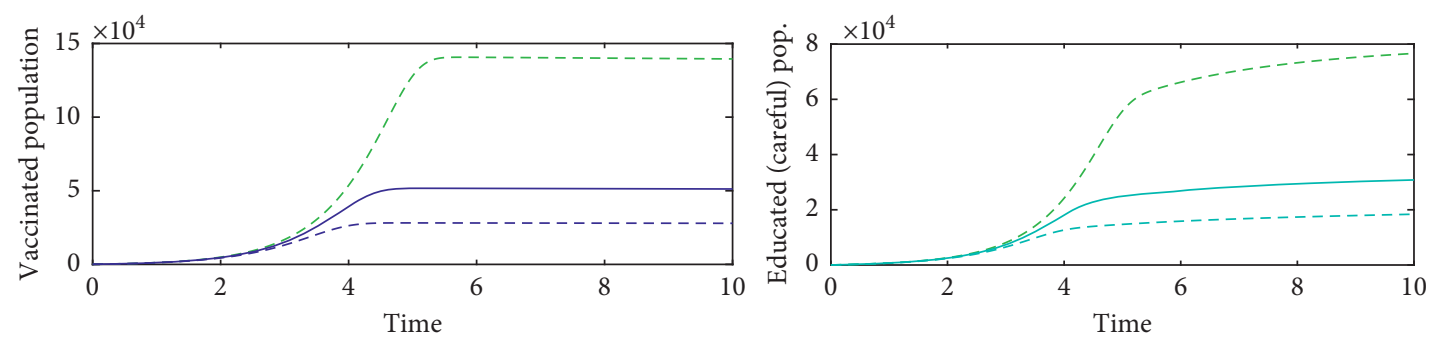

- Rate of infection, $\beta_{1}=0.001$

- Rate of infection, $\beta_{1}=0.002$

- - - Rate of infection, $\beta_{1}=0.003$

Rate of infection, $\beta_{1}=0.001$

Rate of infection, $\beta_{1}=0.002$

- - Rate of infection, $\beta_{1}=0.003$

Figure 9: Trajectories of vaccinated and educated populations for varying rate of infection.
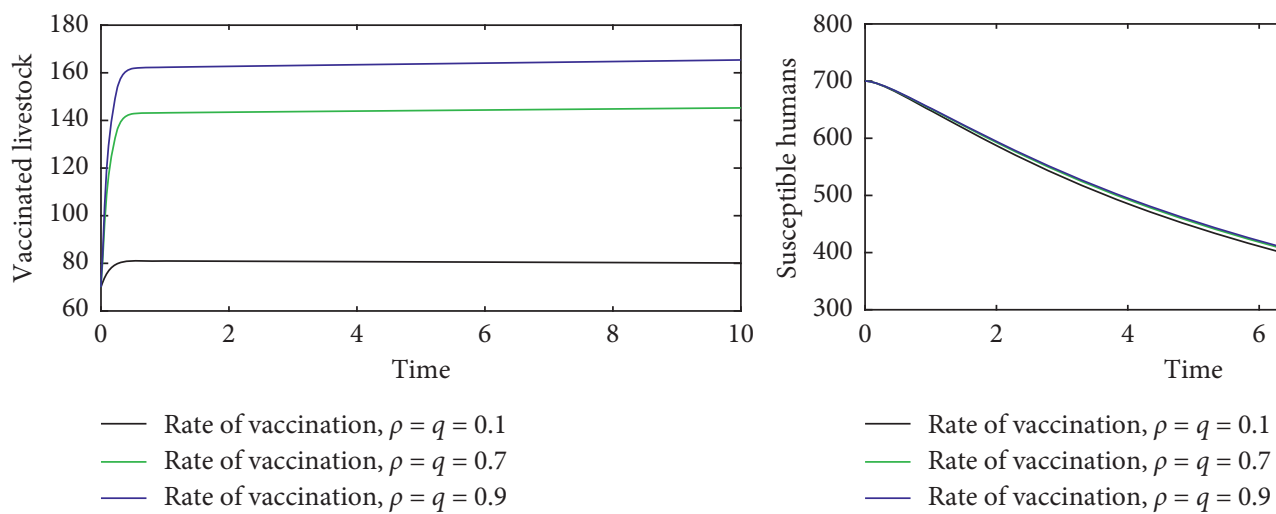

Rate of vaccination, $\rho=q=0.1$
Rate of vaccination, $\rho=q=0.7$
Rate of vaccination, $\rho=q=0.9$

FIgURE 10: Trajectories of susceptible human population for varying rates vaccination.

carcasses, and reducing the level of pathogen in the environment.

Table 4 shows the results of pairing the controls in fighting anthrax disease. The double combinations are ranked according to their efficiency in increasing all susceptible and decreasing all infective populations of both livestock and humans as well as decreasing the number of carcasses and pathogen level in the environment. The most efficient pair in controlling the disease within the livestock population is implementing combined vaccination, and the disinfection program is indicated in (9th row, 1st column) by $R 1$, whereas the most efficient combination pair for the human population is combined vaccination and education indicated in (10th row, 3 rd column) by $R 1$. The overall best pair in the entire model is implementing combined vaccination and education which is 19 (the least value of the total ranks) in the (last row, 3rd column). 

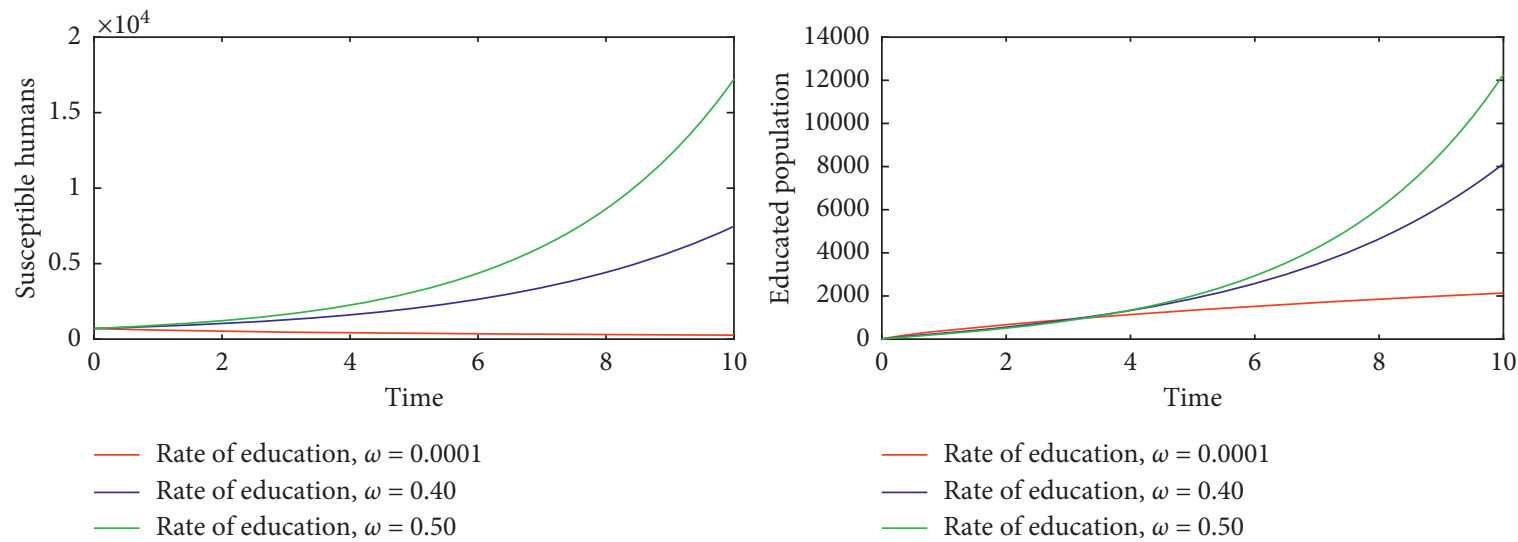

FIgURE 11: Impact of education on susceptible and educated humans.

TABLE 3: Single combination of controls.

\begin{tabular}{|c|c|c|c|c|}
\hline State variables & Vaccination & Education & Disinfection & Treatment \\
\hline Susceptible livestock, $S_{\mathrm{L}}$ & $900(R 3)$ & $1100(R 2)$ & $1900(R 1)$ & $700(R 4)$ \\
\hline Vaccinated livestock, $V_{\mathrm{L}}$ & $1001(R 1)$ & $10(R 2)$ & $10(R 2)$ & $10(R 2)$ \\
\hline Infected livestock, $I_{\mathrm{L}}$ & $1500(R 1)$ & $2700(R 2)$ & $3100(R 4)$ & $2700(R 2)$ \\
\hline Infected carcass, $C$ & $600(R 1)$ & $1100(R 2)$ & $1100(R 2)$ & $1100(R 2)$ \\
\hline Pathogen, $P$ & $1400(R 2)$ & $2300(R 3)$ & $600(R 1)$ & $2300(R 3)$ \\
\hline Susceptible humans, $S_{\mathrm{H}}$ & $700(R 2)$ & $1100(R 1)$ & $700(R 2)$ & $700(R 2)$ \\
\hline Educated humans, $E_{\mathrm{d}}$ & $2100(R 4)$ & $3400(R 1)$ & $2490(R 3)$ & $2600(R 2)$ \\
\hline Infected humans, $I_{\mathrm{H}}$ & $300(R 2)$ & $400(R 3)$ & $600(R 4)$ & $200(R 1)$ \\
\hline Livestock pop. $\left(S_{\mathrm{L}}+V_{\mathrm{L}}-I_{\mathrm{L}}\right)$ & $401(R 1)$ & $-1590(R 2)$ & $-1190(R 3)$ & $-1990(R 4)$ \\
\hline Human pop. $\left(S_{\mathrm{H}}+E_{\mathrm{d}}-I_{\mathrm{H}}\right)$ & $2500(R 4)$ & $4100(R 1)$ & $2590(R 3)$ & $3100(R 2)$ \\
\hline Total rank per control & 21 & 19 & 25 & 24 \\
\hline
\end{tabular}

TABLE 4: Double combination of controls. $\mathrm{V}=$ vaccination, $\mathrm{D}=$ disinfection, $\mathrm{T}=$ treatment, and $\mathrm{E}=$ education.

\begin{tabular}{|c|c|c|c|c|c|c|}
\hline State variables & $\mathrm{V} \& \mathrm{D}$ & $V \& T$ & $\mathrm{~V} \& \mathrm{E}$ & $\mathrm{T} \& \mathrm{D}$ & $T \& E$ & $E \& D$ \\
\hline Susceptible livestock, $S_{\mathrm{L}}$ & $1,100(R 3)$ & $900(R 4)$ & $900(R 4)$ & $1,800(R 1)$ & $1,400(R 2)$ & $1,800(R 1)$ \\
\hline Vaccinated livestock, $V_{\mathrm{L}}$ & $1,500(R 1)$ & $1,001(R 2)$ & $1,001(R 2)$ & $10(R 3)$ & $10(R 3)$ & $10(R 3)$ \\
\hline Infected livestock, $I_{\mathrm{L}}$ & $1,800(R 2)$ & $1,500(R 1)$ & $1,500(R 1)$ & $3,600(R 4)$ & $2,700(R 3)$ & $3,700(R 5)$ \\
\hline Infected carcass, $C$ & $600(R 2)$ & $600(R 2)$ & $600(R 2)$ & $1,200(R 3)$ & $100(R 1)$ & $1,200(R 3)$ \\
\hline Pathogen, $P$ & $400(R 1)$ & $1,400(R 3)$ & $1,400(R 3)$ & $600(R 2)$ & $2,300(R 4)$ & $600(R 2)$ \\
\hline Susceptible humans, $S_{\mathrm{H}}$ & $600(R 4)$ & $600(R 4)$ & $2,600(R 1)$ & $600(R 4)$ & $1,600(R 3)$ & $2,200(R 2)$ \\
\hline Educated humans, $E_{\mathrm{d}}$ & $2,500(R 5)$ & $2,500(R 5)$ & $4,900(R 1)$ & $3,400(R 4)$ & $4,400(R 2)$ & $4,001(R 3)$ \\
\hline Infected humans, $I_{\mathrm{H}}$ & $300(R 2)$ & $10(R 1)$ & $300(R 2)$ & $400(R 3)$ & $10(R 1)$ & $600(R 4)$ \\
\hline Livestock pop. $\left(S_{\mathrm{L}}+V_{\mathrm{L}}-I_{\mathrm{L}}\right)$ & $800(R 1)$ & $401(R 2)$ & $401(R 2)$ & $-1,790(R 4)$ & $-1,290(R 3)$ & $-1,890(R 5)$ \\
\hline Human pop. $\left(S_{\mathrm{H}}+E_{\mathrm{d}}-I_{\mathrm{H}}\right)$ & $2,800(R 6)$ & $3,091(R 5)$ & $7,200(R 1)$ & $3,600(R 4)$ & $5,990(R 2)$ & $5,601(R 3)$ \\
\hline Total rank per control & 27 & 29 & 19 & 33 & 25 & 32 \\
\hline
\end{tabular}

Table 5 shows the implementation of three different controls at the same time. The results show that the most efficient triple combination of controls within the livestock population is implementing combined vaccination, education, and disinfection programme, and this is indicated in (9th row, 3rd column) by $R 1$. The most efficient triple combination for the human population is combining vaccination, education and disinfection as indicated in (10th row, 3rd column) by $R 1$. The overall best triple combination in the entire model is implementing vaccination, education, and disinfection indicated in the (last row, 3rd column) by 12 (the least value of the total ranks).

\section{Conclusions and Recommendations}

5.1. Conclusions. In this work, a nonlinear mathematical model is proposed to study the transmission of anthrax in both livestock and human populations in the presence of vaccination of livestock, education of the human population, treatment of infectives, and disinfection of carcasses and pathogens in the environment.

In order to control the disease from the populations, the parameters such as the pathogen-shedding rate, the anthrax infection rate, the anthrax-induced death rate, and the rate of animals that lose immunity which have the most sensitive positive indexes should be reduced since a reduction in these 
TABle 5: Triple combination of controls. $\mathrm{V}=$ vaccination, $\mathrm{D}=$ disinfection, $\mathrm{T}=$ treatment, and $\mathrm{E}=$ education.

\begin{tabular}{|c|c|c|c|c|}
\hline State variables & $\mathrm{V}, \mathrm{T} \& \mathrm{E}$ & $\mathrm{V}, \mathrm{T} \& \mathrm{D}$ & $\mathrm{V}, \mathrm{E} \& \mathrm{D}$ & E, T \& D \\
\hline Susceptible livestock, $S_{\mathrm{L}}$ & $1,000(R 4)$ & $1,010(R 3)$ & $1,050(R 2)$ & $1,800(R 1)$ \\
\hline Vaccinated livestock, $V_{\mathrm{L}}$ & $1,000(R 2)$ & $1,500(R 1)$ & $1,500(R 1)$ & $10(R 3)$ \\
\hline Infected livestock, $I_{\mathrm{L}}$ & $1,500(R 1)$ & $1,800(R 2)$ & $1,800(R 2)$ & $3,900(R 3)$ \\
\hline Infected carcass, $C$ & $500(R 1)$ & $500(R 1)$ & $500(R 1)$ & $1,000(R 2)$ \\
\hline Pathogen, $P$ & $1,200(R 3)$ & $400(R 1)$ & $400(R 1)$ & $700(R 2)$ \\
\hline Susceptible humans, $S_{\mathrm{H}}$ & $3,100(R 3)$ & $600(R 4)$ & $4,400(R 1)$ & $3,900(R 2)$ \\
\hline Educated humans, $E_{\mathrm{d}}$ & $6,000(R 1)$ & $3,010(R 2)$ & $6,000(R 1)$ & $6,000(R 3)$ \\
\hline Infected humans, $I_{\mathrm{H}}$ & $10(R 1)$ & $10(R 1)$ & $400(R 2)$ & $400(R 2)$ \\
\hline Livestock pop. $\left(S_{\mathrm{L}}+V_{\mathrm{L}}-I_{\mathrm{L}}\right)$ & $500(R 3)$ & $710(R 2)$ & $750(R 1)$ & $-2,090(R 4)$ \\
\hline Human pop. $\left(S_{\mathrm{H}}+E_{\mathrm{d}}-I_{\mathrm{H}}\right)$ & $9,090(R 3)$ & $3,600(R 4)$ & $10,000(R 1)$ & $9,500(R 2)$ \\
\hline Total rank per control & 22 & 21 & 13 & 24 \\
\hline
\end{tabular}

parameters will lead to a decrease in the basic reproduction number. In addition, the most sensitive negative parameters such as the rate of disinfection of the pathogen in the environment, the rate of vaccination of susceptible livestock, and the natural death rate of livestock must be increased in other to control the disease, and an increase in these parameters will lead to a decrease in the basic reproduction number. The numerical simulation of the model confirms the results of the sensitivity analysis.

The study of different control parameters combined to fight the anthrax disease shows that the most efficient single implementation of the control parameters is education while the combination of vaccination and education is the most efficient double implementation of the controls. In addition, implementing the combination of vaccination, education, and disinfection is the best option for triple combination of controls, and lastly, implementing all the controls is an excellent option of controlling the transmission of anthrax in both livestock and human population, and all these options depend solely on the availability of resources.

5.2. Recommendations. The transmission of anthrax can greatly be minimised or possibly be eradicated in the population by ensuring that the reproduction number is less than one. This can be achieved through the following recommendations:

(1) Intensify long-term awareness creation on the disease and the practice of prophylaxes through education of the human population.

(2) Carry out vaccination of newborns and susceptible livestock as well as constant monitoring and continual vaccination of livestock in the vaccinated class, and these will prevent the livestock from losing immunity and becoming susceptible again, thereby reducing the number of susceptible as the vaccinated population increases.

(3) Infected carcass should be thoroughly destroyed and carefully disposed off. In addition, the infected area should be disinfected to kill the pathogens, and these practices will reduce the infected population drastically.

(4) Livestock population should be randomly screened for anthrax infection and the infected animals should be rapidly treated, and this will reduce the pathogen that goes into the environment since anthrax-induced deaths would be reduced.

\section{Data Availability}

The numerical data used to support the findings of this research are included within the article.

\section{Disclosure}

This paper forms part of the Mphil. dissertation of the first author, and the co-authors were the supervisors.

\section{Conflicts of Interest}

The author declares that they have no conflicts of interest.

\section{References}

[1] Food and Agriculture Organisation, The State of Food and Agriculture, FAO, Rome, Italy, 2012.

[2] S. T. Baidoo and H. Yusif, "The effect of smallholder livestock production on income of farm households in northern Ghana," Journal of Science and Technology, vol. 36, no. 3, pp. 8-19, 2016.

[3] Ministry of Food and Agriculture, Ghana Livestock Development Policy and Strategy, Ministry of Food and Agriculture, Accra, Ghana, 2016.

[4] African Development Fund, Appraisal Report: Livestock Development Project, African Development Fund, Tunis, Tunisia, 2001.

[5] Ghana Statistical Service, Ghana Living Standard Survey Report of the Fifth Round, Ghana Statistical Service, Accra, Ghana, 2008.

[6] T. P. Robinson, Global Livestock Production Systems, Food and Agriculture Organisation of the United Nations and International Livestock Research Institute, Rome, Italy, 2011.

[7] World Health Organisation, Anthrax in Humans and Animals, P. Turnbull, Ed., WHO Press, Geneva, Switzerland, 4th edition, 2008.

[8] K. Hampson, T. Lembo, P. Bessell et al., "Predictability of anthrax infection in the Serengeti, Tanzania," Journal of Applied Ecology, vol. 48, pp. 1333-1344, 2011.

[9] World Health Organisation, Emerging Infectious Diseases and Zoonoses, WHO, New Delhi, India, 2014.

[10] World Health Organisation, The Control of Neglected Zoonotic Diseases: From Advocacy to Action, Report of the Fourth 
International Meeting Held at WHO Headquaters, World Health Organisation, Geneva, Swtzerlan, 2015.

[11] Food and Agriculture Organisation of the United Nations, Anthrax Outbreaks: a Warning for Improved Prevention, Conrol and Heightened Awareness, Empres Watch, Vol. 37, Food and Agriculture Organisation of the United Nations, Rome, Italy, 2016.

[12] H. W. Hethcote, "The mathematics of infectious diseases," SIAM Review, vol. 42, no. 4, pp. 599-653, 2000.

[13] B. D. Hahn and P. R. Furniss, "A deterministic model of an anthrax epizootic: threshold results," Ecological Modelling, vol. 20, no. 2-3, pp. 233-241, 1983.

[14] A. Friedman and A. Yakubu, "Anthrax epizootic and migration: persistence or extinction," Mathematical Biosciences, vol. 241, no. 1, pp. 137-144, 2013.

[15] S. Mushayabasa, "Global stability of an anthrax model with environmental decontamination and time delay," Discrete Dynamics in Nature and Society, vol. 2015, Article ID 573146, 6 pages, 2015.

[16] Z. M. Sinkie and N. S. Murthy, "Modeling and simulation study of anthrax attack on environment," Journal of Multidisciplinary Engineering Science and Technology, vol. 3, no. 4, pp. 4574-4578, 2016.

[17] R. Kumar, C. C. Chow, J. D. Bartels, G. Clermont, and Y. Vodovozt, "A mathematical simulation of the inflammatory response to anthrax infection," Shock, vol. 29, no. 1, pp. 104-111, 2008.

[18] S. Osman, O. D. Makinde, and M. D. Theuri, "Mathematical modelling of the transmission dynamics of anthrax in human and animal population," Mathematical Theory and Modeling, vol. 8, no. 6, 2018.

[19] V. Lakshmikanthan, S. Leela, and A. A. A. Martyniuk, Stability Analysis of Nonlinear Systems, CRC Press, Boca Raton, FL, USA, 1989.

[20] O. Diekmann, J. A. P. Heesterbeek, and J. Metz, "On the definition and the computation of the basic mathematical biology," Journal of Mathematical Biology, vol. 28, no. 4, pp. 365-382, 1990.

[21] B. Seidu, O. D. Makinde, and I. Y. Seini, "Mathematical analysis of the effects of HIV-malaria co-infection on workplace productivity," Acta Biotheoretica, vol. 63, no. 2, pp. 151-824, 2015.

[22] C. S. Bornaa, Y. I. Seini, and B. Seidu, "Modelling zoonotic diseases with treatment in both human and animal populations," Communications in Mathematical Biology and Neuroscience, vol. 2017, p. 11, 2017. 